\title{
An Ultra-Low Power InAs/AlSb HEMT Ka-Band Low-Noise Amplifier
}

Jonathan B. Hacker, Member, IEEE, Joshua Bergman, Member, IEEE, Gabor Nagy, Gerard Sullivan, Christoph Kadow, Heng-Kuang Lin, A. C. Gossard, Mark Rodwell, Fellow, IEEE, and Berinder Brar, Member, IEEE

\begin{abstract}
The first antimonide-based compound semiconductor (ABCS) MMIC, a Ka-Band low-noise amplifier using 0.25- $\mu \mathrm{m}$ gate length InAs/AISb metamorphic HEMTs, has been fabricated and characterized on a $75 \mu \mathrm{m}$ GaAs substrate. The compact $1.1 \mathrm{~mm}^{2}$ three-stage Ka-band LNA demonstrated an average of $2.1 \mathrm{~dB}$ noise-figure between 34-36 GHz with an associated gain of $22 \mathrm{~dB}$. The measured dc power dissipation of the ABCS LNA was an ultra-low $1.5 \mathrm{~mW}$ per stage, or $4.5 \mathrm{~mW}$ total. This is less than one-tenth the dc power dissipation of a typical equivalent InGaAs/AIGaAs/GaAs HEMT LNA. Operation with degraded gain and noise figure at $1.1 \mathrm{~mW}$ total dc power dissipation is also verified. These results demonstrate the outstanding potential of ABCS HEMT technology for mobile and space-based millimeter-wave applications.
\end{abstract}

Index Terms-Antimonide-based compound semiconductor (ABCS) HEMT, InAs/AISb HFET, low noise amplifier, millimeter-waves.

\section{INTRODUCTION}

$\mathbf{U}$ LTRA-LOW power millimeter-wave low-noise amplifiers (LP-LNAs) represent a critical component for many emerging systems applications including active-array space-based radar and mobile millimeter-wave communications and handheld imagers. For such applications, antimonide-based compound semiconductor (ABCS) InAs/AlSb HEMTs are particularly promising because of their combination of high electron mobility and peak velocity, along with high electron concentration in the 2DEG that results in unparalleled speed-power performance. The InAs/AlSb HEMT's inherent low-voltage operation, with $V_{\mathrm{ds}}$ below $0.5 \mathrm{~V}$, can reduce dc power dissipation by an order of magnitude compared with a GaAs PHEMT of equivalent performance [1], [2], and by a factor of three to four compared to an equivalent InP HEMT [3], [4]. In the case of active-array space-based radar applications, ABCS LP-LNAs are a system enabler because they permit a substantial reduction in the required spacecraft prime power and corresponding spacecraft weight and launch cost [5], [6]. For example, it is estimated that a 200000 -element array

Manuscript received May 31, 2003; revised October 20, 2003. This work was supported by SPAWAR under Contract N66001-01-C-8032, D. Mullin and C. Hanson Program Managers, and by J. Shah, Program Manager, at the Defense Advanced Research Projects Agency (DARPA) Microsystems Technology Office.

J. B. Hacker, J. Bergman, G. Nagy, G. Sullivan, and B. Brar are with the Rockwell Scientific Company, Thousand Oaks, CA 91360 USA (e-mail: jbhacker@ieee.org; jhacker@rwsc.com).

C. Kadow, H.-K. Lin, A. C. Gossard, and M. Rodwell are with the Department of Electrical and Computer Engineering, University of California, Santa Barbara, CA 93106 USA.

Digital Object Identifier 10.1109/LMWC.2004.827132

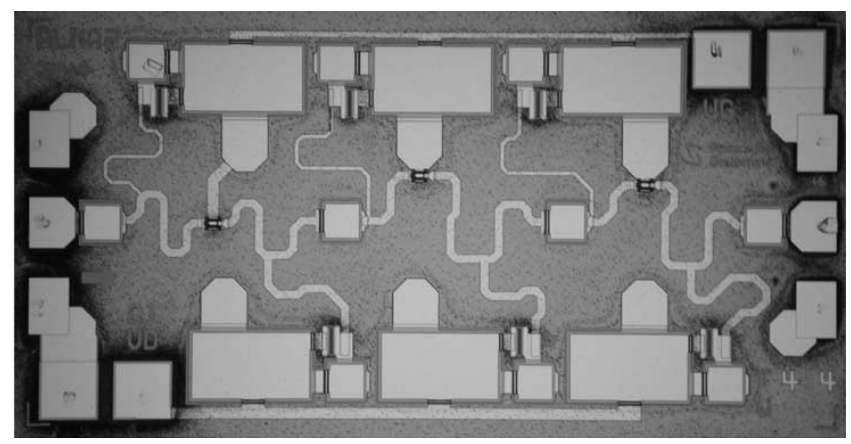

Fig. 1. Photomicrograph of the three-stage ABCS HEMT MMIC Ka-band LNA. The compact die measures $1.5 \mathrm{~mm}$ by $0.7 \mathrm{~mm}$ with a thickness of $75 \mu \mathrm{m}$.

using ABCS LP-LNAs will require $1.6 \mathrm{~kW}$ less prime power compared with a system using GaAs LNAs. The corresponding estimated 1100-pound decrease in launch weight could potentially reduce launch costs by many tens of millions of dollars per spacecraft.

We report here the first ABCS MMIC (Fig. 1). The ultra-low power three-stage LP-LNA operates at Ka-band and uses 0.25 - $\mu$ m-gate-length ABCS HEMT devices. The first pass success for this design is primarily due to a stable ABCS HEMT MMIC technology, high-quality ABCS epitaxial material, accurate active and passive models, and a proven design approach.

\section{Device Design And Characterization}

The ABCS InAs/AlSb HEMT structure (Fig. 2) is grown using molecular beam epitaxy on two-inch and four-inch semi-insulating GaAs substrates using an AlSb buffer to accommodate the lattice mismatch. Tellurium planar doping is employed in the AlSb to simultaneously achieve a high-channel aspect ratio for the $0.25-\mu \mathrm{m}$ gate-length HEMT and high electron transfer efficiency. Hall measurements show sheet carrier concentration and mobility to be $3.7 \times 10^{12} \mathrm{~cm}^{-2}$ and $19000 \mathrm{~cm}^{2} / \mathrm{Vs}$ at $295 \mathrm{~K}$. The corresponding values at $77 \mathrm{~K}$ are $3.4 \times 10^{12} \mathrm{~cm}^{-2}$ and $65000 \mathrm{~cm}^{2} / \mathrm{Vs}$ and are indicative of the excellent quality of the MBE grown material. The device is capped with a strained InAlAs layer to provide a chemically stable surface layer and minimize gate leakage effects [7].

The 0.25- $\mu \mathrm{m}$-gate-length ABCS HEMT has measured dc transconductance of $1.5 \mathrm{~S} / \mathrm{mm}$ and $2.0 \mathrm{~S} / \mathrm{mm}$ at $V_{\mathrm{ds}}$ of $0.3 \mathrm{~V}$ and $0.4 \mathrm{~V}$ respectively and $\mathrm{I}_{\mathrm{dss}}$ is $1 \mathrm{~A} / \mathrm{mm}$ at $V_{\mathrm{ds}}$ of $0.4 \mathrm{~V}$. For a $2 \times 20 \mu \mathrm{m}$ device, on-wafer $s$-parameter measurements show a cutoff frequency, $f_{\tau}$, of $160 \mathrm{GHz}$ and a maximum oscillation frequency, $f_{\max }$, of $190 \mathrm{GHz}$. The $f_{\max }$ is improved 


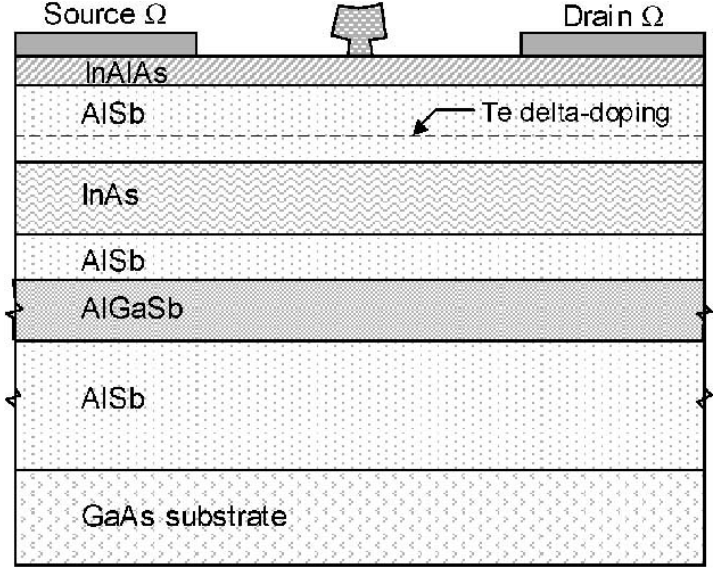

Fig. 2. Layer structure of an antimonide-based compound semiconductor (ABCS) InAs/AlSb HEMT grown by MBE on semi-insulating 2-in and 4-in diameter GaAs substrates.

significantly over published results, but increasing $f_{\max }$ further presents a challenge, as it is likely a result of high input and output conductances due to the narrow bandgap of InAs. The devices do not show a well-defined knee voltage due to a high relative threshold voltage and lack of velocity saturation at fields typically present in the InAs channel. Soft breakdown is observed at drain voltages above $0.4 \mathrm{~V}$ and reflects the onset of impact ionization in the channel. Further details on the ABCS HEMT structure and its performance as used in this MMIC have been reported elsewhere [8].

\section{AMPLIFIER DESIGN AND FABRICATION}

The three-stage Ka-band LP-LNA was designed using $0.25 \mu \mathrm{m}$ gate length two-finger, $40-\mu \mathrm{m}$ total gate width ABCS HEMTs. The linear small-signal model used for the design was based upon small-signal $s$-parameter measurements to $50 \mathrm{GHz}$, and noise parameter measurements to $26 \mathrm{GHz}$. Device pad parasitics were carefully deembedded from the raw measurements. The noise model parameters are obtained by fitting to the measured noise parameters from 2 to $26 \mathrm{GHz}$, and then extrapolating the model to Ka-band. The devices are biased at a nominal drain voltage of $0.35 \mathrm{~V}$ and $4.5 \mathrm{~mA}$ of drain current. At $35 \mathrm{GHz}$, the modeled device noise parameters are $\mathrm{NF}_{\text {min }}=1.46 \mathrm{~dB}, \Gamma_{\mathrm{opt}}=0.592 \angle 58.6^{\circ}$ and $\mathrm{R}_{\mathrm{n}}=23 \Omega$.

The $2 \times 20 \mu \mathrm{m}$ ABCS HEMTs used in the amplifier are interconnected with microstrip matching networks. Air-bridges were used for the device source connections. The first stage device employs inductive feedback in the source to simultaneously optimize input impedance and noise match. Epitaxial resistors and SiN MIM capacitors were used for the bias network and interstage dc blocks. The semi-insulating GaAs substrate was thinned to $75 \mu \mathrm{m}$ and through-substrate vias dry etched from the back.

The ABCS HEMT MMIC fabrication process was derived from Rockwell Scientific's production GaAs PHEMT process [1]. Following this approach minimized development risk and improved the chance of first-pass success for the ABCS MMIC.

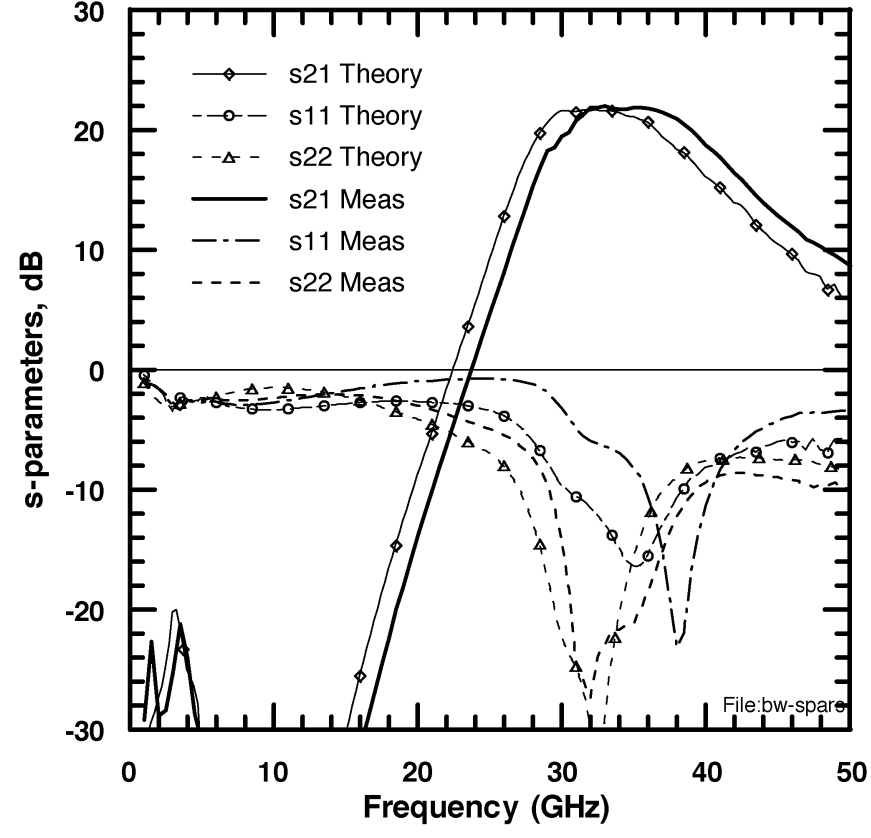

Fig. 3. Measured insertion gain (s21) and input (s11) and output (s22) return loss of the ABCS LP-LNA compared with the theoretical prediction from circuit model. The LP-LNA is unconditionally stable across the measured frequency range.

The main differences between the two processes are found in the isolation and ohmic contact steps, and the electron-beam lithography process for defining the gate.

\section{AMPLIFIER CHARACTERIZATION}

Performance of the three-stage amplifier was measured on-wafer with GGB Picoprobe rf probes. The measured $s$-parameters are shown in Fig. 3 where they are compared with simulated results. The agreement between theory and measurement is close, with an observed $1 \mathrm{GHz}$ shift upwards in frequency of the measured gain bandwidth. The shift is also observed in the input match, suggesting gate length variation may be the principle cause. Over the band from 34 to $36 \mathrm{GHz}$, the measured average gain is $21.8 \mathrm{~dB}$, the input match is better than $8 \mathrm{~dB}$, and the output match is better than $18 \mathrm{~dB}$. The amplifier was unconditionally stable with $\mathrm{K}>2.6$ over the measured frequency range up to $50 \mathrm{GHz}$. Noise figure measurements are shown in Fig. 4 along with simulated results. The measured amplifier average noise figure over the band from 34 to $36 \mathrm{GHz}$ is $2.1 \mathrm{~dB}$, which agrees closely with the simulated result of $2.2 \mathrm{~dB}$. Load pull measurements at $8 \mathrm{GHz}$ show the output power of a discrete $2 \times 20 \mu \mathrm{m}$ device at $V_{\mathrm{ds}}=0.35 \mathrm{~V}$ is $-4.7 \mathrm{dBm}$ at the $1 \mathrm{~dB}$ gain compression point.

The ABCS HEMT has a very low drain saturation voltage, $\mathrm{V}_{\mathrm{dsat}}$, where electrons in the channel reach peak drift velocity. Consequently, the LP-LNA can be biased at exceptionally low drain voltages where dissipated dc power is extremely small. For lowest noise figure, the rf measurements were made with $V_{\mathrm{dd}}=0.35 \mathrm{~V}$ and $I_{\mathrm{d}}=12.8 \mathrm{~mA}$, corresponding to a de power of $4.5 \mathrm{~mW}$. This dc power dissipation is less than one-tenth the power consumption of Ka-band amplifiers using GaAs-based HEMTs that are typically biased at $2 \mathrm{~V}$ or more, and one-third that of InP-based HEMTs that are typically biased at $0.75 \mathrm{~V}$ or 


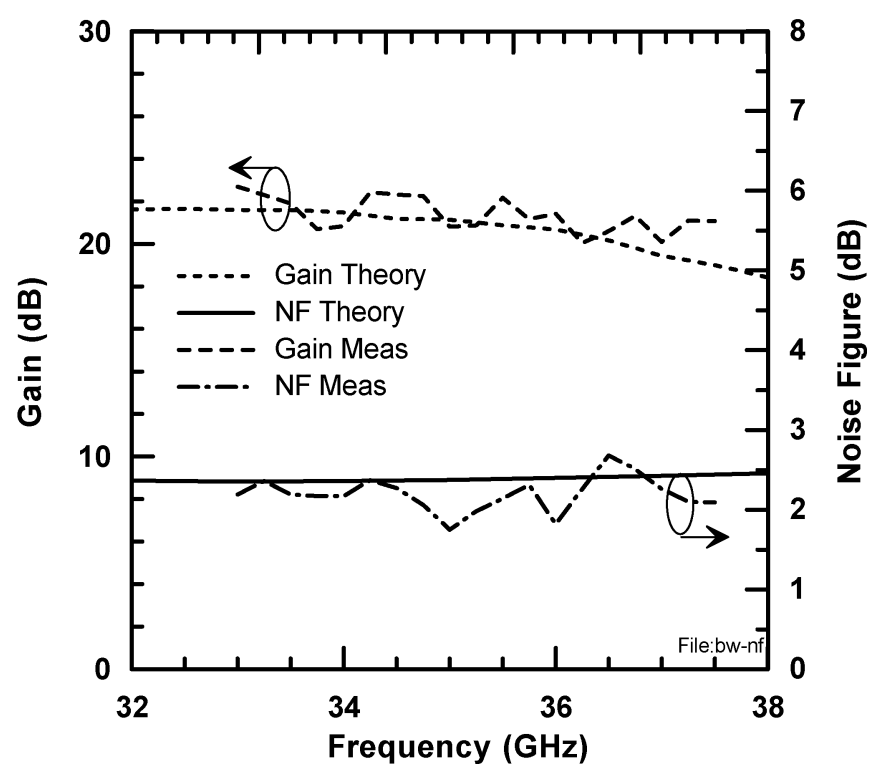

Fig. 4. Measured noise figure and associated gain of the ABCS LP-LNA compared with the theoretical prediction from circuit model confirms the repeatability of the ABCS MMIC process.

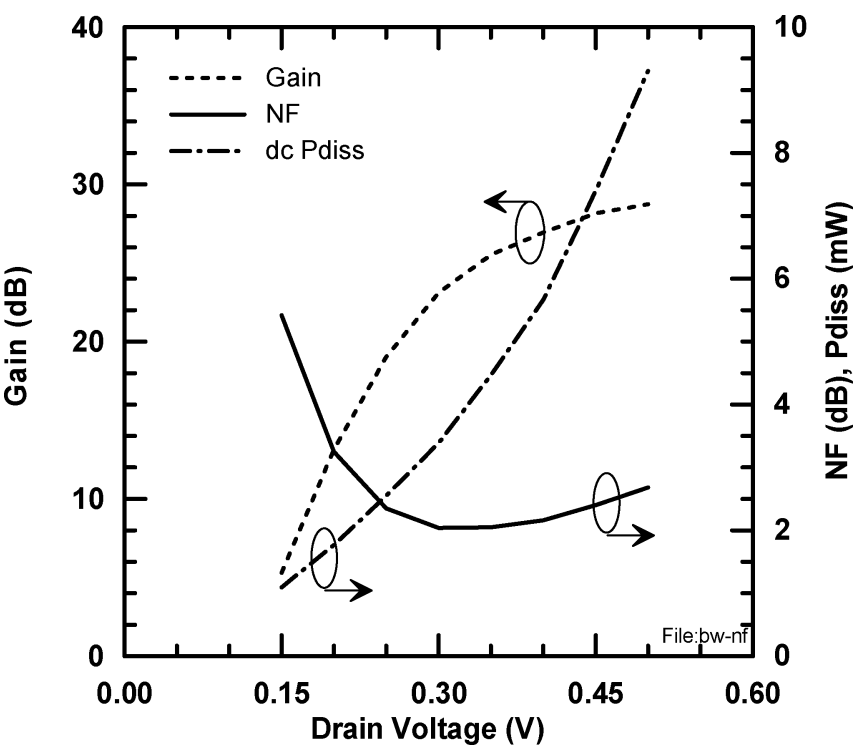

Fig. 5. Plot of measured gain and noise figure at $35 \mathrm{GHz}$, and total dc power dissipation versus applied LP-LNA drain voltage. Operation with dc power dissipation as low as $1.1 \mathrm{~mW}$ is demonstrated with degraded gain and noise figure.

more. Fig. 5 shows the gain, noise figure, and dc power dissipation at $35 \mathrm{GHz}$ for a range of drain voltages from 0.15 to $0.50 \mathrm{~V}$. For some applications where operation with degraded gain and noise figure may be tolerated, operating the amplifier as low as $V_{\mathrm{dd}}=0.15 \mathrm{~V}$ leads to a dc power dissipation of only $1.1 \mathrm{~mW}$, which is believed to be the lowest reported dc power dissipation of any millimeter-wave low noise amplifier.

\section{CONCLUSION}

A three-stage ABCS HEMT ultra-low-power low-noise amplifier (LP-LNA) designed to operate at Ka-Band is reported. The amplifier achieved $22 \mathrm{~dB}$ gain and $2.1 \mathrm{~dB}$ average noise figure from 34 to $36 \mathrm{GHz}$ with a total dc power dissipation of only $4.5 \mathrm{~mW}$ using relatively long $0.25 \mu \mathrm{m}$ gate length devices. Decreasing the device gate length to $0.1 \mu \mathrm{m}$ is expected to produce a new class of amplifiers with even lower power dissipation while capable of operation at frequencies up to W-band and higher. These results demonstrate the outstanding potential of ABCS HEMT technology for mobile and space-based millimeter-wave applications.

\section{ACKNOWLEDGMENT}

The authors thank D. Deakin, D. Regan, A. Paniagua, P. Hundal, S. Pittman, C. Regan, J. Greer, L. Tran, and A. Ikhlassi for device fabrication.

\section{REFERENCES}

[1] Y. Kwon, D. S. Deakin, E. A. Sovero, and J. A. Higgins, "High-performance Ka-band monolithic low-noise amplifiers using $0.2-\mu \mathrm{m}$ dry-recessed GaAs PHEMTs," IEEE Microwave Guided Wave Lett., vol. 6, pp. 253-255, July 1996.

[2] E. Heaney, F. McGrath, P. O'Sullivan, and C. Kermarrec, "Ultra low power low noise amplifiers for wireless communications," in $1993 \mathrm{GaAs}$ IC Symp. Tech. Dig., Oct. 1993, pp. 49-51.

[3] D. C. W. Lo, R. Lai, H. Wang, K. L. Tan, R. M. Dia, D. C. Streit, P.-H. Liu, J. Velebir, B. Allen, and J. Berenz, "A high-performance monolithic Q-band InP-based HEMT low-noise amplifier," IEEE Microwave Guided Wave Lett., vol. 3, no. 9, pp. 299-301, Sept. 1993.

[4] R. Lai, K. W. Chang, H. Wang, K. Tan, D. C. Lo, D. C. Streit, P. H. Liu, R. Dia, and J. Berenz, "A high performance and low DC power V-band MMIC LNA using $0.1 \mu \mathrm{m}$ InGaAs/InAlAs/InP HEMT technology," IEEE Microwave Guided Wave Lett., vol. 3, pp. 447-449, Dec. 1993.

[5] S. Fiedler and B. Preiss, "Geosynchronous space based radar concept development for theater surveillance," in 1996 IEEE Aerospace Applications Conf. Proc., vol. 4, Feb. 1996, pp. 77-90.

[6] M. E. Davis, "Space based radar core technology challenges for affordability," in 2001 Core Technologies for Space Systems Conference Dig., Colorado Springs, CO, Nov. 2001.

[7] J. B. Boos, W. Kruppa, B. R. Bennett, D. Park, S. W. Kirchoefer, R. Bass, and H. B. Dietrich, "AlSb/InAs HEMT's for low-voltage, high-speed applications," IEEE Trans. Electron Devices, vol. 45, pp. 1869-1875, Sept. 1998

[8] J. Bergman, G. Nagy, G. Sullivan, B. Brar, C. Kadow, H.-K. Lin, A. Gossard, and M. Rodwell, "InAs/AlSb HFET's with $\mathrm{ft}$ and fmax above $150 \mathrm{GHz}$ for low-power MMICs," in 2003 Intl. Conf. InP and Related Materials Proc., May 2003, pp. 219-222. 